TITLE:

\title{
Mechanism of superconductivity in a two-dimensional double- exchange system with spin-orbit coupling
}

$\operatorname{AUTHOR}(\mathrm{S})$ :

Morinari, $T$

CITATION:

Morinari, T. Mechanism of superconductivity in a two-dimensional double-exchange system with spin-orbit coupling. PHYSICAL REVIEW B 2001, 64(1): 012510.

ISSUE DATE:

2001-07-01

URL:

http://hdl.handle.net/2433/50468

RIGHT:

Copyright 2001 American Physical Society 
PHYSICAL REVIEW B, VOLUME 64, 012510

\title{
Mechanism of superconductivity in a two-dimensional double-exchange system with spin-orbit coupling
}

\author{
Takao Morinari \\ Yukawa Institute for Theoretical Physics, Kyoto University, Kyoto 606-8502, Japan
}

(Received 22 January 2001; published 15 June 2001)

\begin{abstract}
We propose a mechanism of unconventional superconductivity in a two-dimensional double-exchange system with spin-orbit coupling. We show that a Chern-Simons term is induced by integrating out the conductionelectron fields. In the presence of the Chern-Simons term, the conduction electrons behave like skyrmion or antiskyrmion excitations for the core spin system, which leads to a disordered state of the core spins. After magnetic long-range order is destroyed, the Chern-Simons term becomes dominant for the low-energy physics and leads to a $p$-wave pairing state of conduction electrons.
\end{abstract}

DOI: $10.1103 /$ PhysRevB.64.012510

PACS number(s): 74.20.Mn, 74.80.Dm, 75.10.Jm

The occurrence of unconventional superconductivity in strongly correlated electron systems is one of the fascinating problems in condensed-matter physics. A typical candidate is the set of high- $T_{c}$ cuprates. Superconductivity of cuprates occurs on a hole-doped antiferromagnetic insulator. ${ }^{1}$ The undoped parent compound has a layered structure of $\mathrm{CuO}_{2}$ planes and is a Mott insulator with spins at $\mathrm{Cu}$ sites antiferromagnetically ordered. Upon doping with holes in the $\mathrm{CuO}_{2}$ plane, antiferromagnetic long-range order is destroyed and $d$-wave superconductivity emerges. It seems that the occurrence of superconductivity in high- $T_{c}$ cuprates is based on a disordered spin background and the layered structure of $\mathrm{CuO}_{2}$ plane is essential for the occurrence of superconductivity.

Superconductivity in $\mathrm{Sr}_{2} \mathrm{RuO}_{4},{ }^{2}$ which has the same crystal structure as $\mathrm{La}_{2} \mathrm{CuO}_{4}$, also occurs near magnetic ordering. $\mathrm{Sr}_{2} \mathrm{RuO}_{4}$ has a layered structure of $\mathrm{RuO}_{2}$ plane instead of $\mathrm{CuO}_{2}$ plane. It belongs to the Ruddlesen-Popper series $\mathrm{Sr}_{n+1} \mathrm{Ru}_{n} \mathrm{O}_{3 n+1}$, which are multilayered compounds with $n$ the number of $\mathrm{RuO}_{2}$ planes per unit cell. With increasing $n$ a ferromagnetic correlation becomes large and the $n=\infty$ compound $\mathrm{SrRuO}_{3}$ has three-dimensional ferromagnetic order. ${ }^{3}$ For $n=1$, which is the case of $\mathrm{Sr}_{2} \mathrm{RuO}_{4}$, there is no ferromagnetic order but it seems to be near ferromagnetic order., From this observation, superconductivity of $\mathrm{Sr}_{2} \mathrm{RuO}_{4}$ was proposed to be a $p$-wave pairing state using the analogy to ${ }^{3}$ He system. ${ }^{6,7}$ Moreover, increase of three dimensionality, or the ferromagnetic correlation apparently destroys superconductivity, because the $n=2$ compound $\mathrm{Sr}_{3} \mathrm{Ru}_{2} \mathrm{O}_{7}$ does not exhibit superconductivity. ${ }^{8}$ It seems that the layered structure is also essential for superconductivity of $\mathrm{Sr}_{2} \mathrm{RuO}_{4}$ and superconductivity is based on a disordered spin background.

Although there are many discrepancies between the high$T_{c}$ cuprates and the $\mathrm{Sr}_{2} \mathrm{RuO}_{4},{ }^{5}$ above arguments suggest that the layered structure and the disordered spin background are the key to understand superconductivity of these systems.

The purpose of this Report is to propose a model of unconventional superconductivity that is based on a purely two-dimensional mechanism and a disordered spin background. We consider a two-dimensional double-exchange system with spin-orbit coupling and assume a ferromagnetic correlation between core spins for simplicity. We perform a SU(2) transformation at each site so that Hund coupling has a diagonal form. The gauge field, which describes the fluctuation of the core spins, is introduced in this process. By the derivative expansion we show that the Chern-Simons term is induced after the conduction-electron fields are integrated out. The conduction electrons are related to skyrmion or antiskyrmion excitations depending on their spin through the Chern-Simons term. A conduction electron with $\uparrow(\downarrow)$ spin induces a skyrmion (antiskyrmion) in the spin system. These skyrmion excitations introduce disorder for the core spins. If they destroy ferromagnetic long-range order, the ChernSimons term becomes dominant for the low-energy physics. For the conduction electrons, the Chern-Simons term leads to a $p$-wave pairing state. The pairing mechanism is similar to that found by Greiter, Wen, and Wilczek in the $\nu=1 / 2$ quantum-Hall system. ${ }^{9}$ Contrary to anyon superconductivity, ${ }^{10}$ quasiparticles do not obey the semion statistics because the Chern-Simons term does not alter the statistics of particles.

The Hamiltonian for a two-dimensional double-exchange system with spin-orbit coupling is given by

$$
H=-t_{0} \sum_{\langle i, j\rangle}\left(c_{i}^{\dagger} c_{j}+\text { H.c. }\right)-J_{\mathrm{H}} \sum_{j} \mathbf{s}_{j} \cdot \mathbf{S}_{j}^{c}+H_{\mathrm{so}}+H_{\mathrm{spin}},
$$

where

$$
c_{i}^{\dagger}=\left(\begin{array}{ll}
c_{i \uparrow}^{\dagger} & c_{i \downarrow}^{\dagger}
\end{array}\right) \quad \text { and } \quad c_{i}=\left(\begin{array}{c}
c_{i \uparrow} \\
c_{i \downarrow}
\end{array}\right)
$$

denote a creation and an annihilation operator for the conduction-electron field in a spinor representation. For the first term, the summation is taken over the nearest-neighbor sites. The second term denotes Hund coupling between the conduction electron spin $\mathbf{s}_{j}=\frac{1}{2} c_{j}^{\dagger} \boldsymbol{\sigma} c_{j}$, and the core $\operatorname{spin} \mathbf{S}_{j}^{c}$. For spin-orbit coupling, we assume the following form:

$$
H_{\mathrm{so}}=i \sum_{j} \sum_{\alpha=x, y} c_{j}^{\dagger} \boldsymbol{\lambda}^{(\alpha)} \cdot \boldsymbol{\sigma} c_{j+a \hat{e}_{\alpha}}+\text { H.c., }
$$


where $\boldsymbol{\lambda}^{(\alpha)}=\left(\lambda_{x}^{(\alpha)}, \lambda_{y}^{(\alpha)}\right)$ and $\hat{e}_{\alpha}$ is a unit vector along $\alpha$ axis and $a$ is the lattice constant(we consider the square lattice for simplicity). Spin-orbit coupling given by Eq. (2) is based on a multiband model. ${ }^{11,12}$ In the presence of spin-orbit coupling Eq. (2), there is generally a Dzyaloshinskii-Moriya type interaction ${ }^{13}$ for the core spin system. However, such term does not affect the following argument. We assume that the core spins are ferromagnetically coupled and described by the Hamiltonian: $H_{\text {spin }}=-J \Sigma_{\langle i, j\rangle} \mathbf{S}_{i}^{c} \cdot \mathbf{S}_{j}^{c}$. In order to describe the core spins, we introduce the Schwinger bosons: ${ }^{14} \mathbf{S}_{j}^{c}$ $=\frac{1}{2} z_{j}^{\dagger} \boldsymbol{\sigma} z_{j}$ with

$$
z_{j}^{\dagger}=\left(\begin{array}{ll}
z_{j \uparrow}^{\dagger} & z_{j \downarrow}^{\dagger}
\end{array}\right) \quad \text { and } \quad z_{j}=\left(\begin{array}{c}
z_{j \uparrow} \\
z_{j \downarrow}
\end{array}\right) .
$$

Here $z_{j \sigma}$ are boson fields and obey the constraint $z_{j \uparrow}^{\dagger} z_{j \uparrow}$ $+z_{j \downarrow}^{\dagger} z_{j \downarrow}=2 S$.

We turn to the path-integral formulation to describe the system. The action is given by

$$
S=\int d t\left\{\sum_{j}\left[\bar{c}_{j}(t) i \partial_{t} c_{j}(t)+\bar{z}_{j}(t) i \partial_{t} z_{j}(t)\right]-H\right\} .
$$

In order to describe the fluctuation of the core spins, we introduce a gauge field. The effect of the gauge field to the conduction electrons can be introduced by performing a series of $\mathrm{SU}(2)$ transformations. Let us focus on an $i-j$ bond along $\alpha$ axis where $i$ and $j$ are nearest-neighbor sites. The hopping term is given by

$$
h_{i j}\left(\boldsymbol{\lambda}^{(\alpha)}\right)=\sqrt{t_{0}^{2}+\lambda_{\alpha}^{2}} \bar{c}_{i} \chi\left(\boldsymbol{\lambda}^{(\alpha)}\right) c_{j},
$$

where $\chi\left(\boldsymbol{\lambda}^{(\alpha)}\right)=1 / \sqrt{t_{0}^{2}+\lambda_{\alpha}^{2}}\left(-t_{0} \sigma_{0}+i \boldsymbol{\lambda}^{(\alpha)} \cdot \boldsymbol{\sigma}\right)$, with $\sigma_{0}$ the unit matrix in spin space and $\lambda_{\alpha}=\left|\boldsymbol{\lambda}^{(\alpha)}\right|$. For the case of $\lambda_{\alpha} \neq 0$, there is a twist in spin space. To begin with, we perform a transformation to diagonalize the hopping term,

$$
c_{i} \rightarrow \chi^{\dagger}\left(\boldsymbol{\lambda}^{(\alpha)} / 2\right) c_{i}, \quad \bar{c}_{j} \rightarrow \bar{c}_{j} \chi^{\dagger}\left(\boldsymbol{\lambda}^{(\alpha)} / 2\right),
$$

By this transformation, we obtain $h_{i j}\left(\boldsymbol{\lambda}^{(\alpha)}\right)=$ $-\sqrt{t_{0}^{2}+\lambda_{\alpha}^{2}} \bar{c}_{j} c_{i}$, up to $O\left(\left(\lambda_{\alpha} / t\right)^{3}\right)$. Second, we perform a transformation which diagonalizes Hund coupling term: $c_{i}$ $\rightarrow U_{i} c_{i}, \bar{c}_{j} \rightarrow \bar{c}_{j} \bar{U}_{j}$, where

$$
U_{j}=\left(\begin{array}{cc}
z_{j \uparrow} & -\bar{z}_{j \downarrow} \\
z_{j \downarrow} & \bar{z}_{j \uparrow}
\end{array}\right) \quad \text { and } \quad \bar{U}_{j}=\left(\begin{array}{cc}
\bar{z}_{j \uparrow} & \bar{z}_{j \downarrow} \\
-z_{j \downarrow} & z_{j \uparrow}
\end{array}\right) .
$$

By this transformation, SU(2)-gauge fields that describe the fluctuation of the core spins are introduced. We set

$$
\begin{aligned}
\bar{U}_{i} U_{j} & =\left(\begin{array}{cc}
\bar{z}_{i \uparrow} z_{j \uparrow}+\bar{z}_{i \downarrow} z_{j \downarrow} & -\bar{z}_{i \uparrow} \bar{z}_{j \downarrow}+\bar{z}_{i \downarrow} \bar{z}_{j \uparrow} \\
z_{i \uparrow} z_{j \downarrow}-z_{i \downarrow} z_{j \uparrow} & z_{i \uparrow} \bar{z}_{j \uparrow}+z_{i \downarrow} \bar{z}_{j \downarrow}
\end{array}\right) \\
& \equiv\left|\bar{U}_{i} U_{j}\right| \exp \left(-i a \mathcal{A}_{j i}\right) .
\end{aligned}
$$

Since the amplitude fluctuation of the core spins: $\left|\bar{U}_{i} U_{j}\right|$, is relatively high-energy mode than the phase fluctuation of that, we take its mean value $\eta=\left\langle\left|\bar{U}_{i} U_{j}\right|\right\rangle$. Equation (6) is the definition of the lattice $\mathrm{SU}(2)$-gauge field $\mathcal{A}_{j i} \cdot{ }^{15}$ Finally, we perform the inverse transformation of Eq. (5). As a result, we obtain

$$
h_{i j}\left(\boldsymbol{\lambda}^{(\alpha)}\right)=-\sqrt{t_{0}^{2}+\lambda_{\alpha}^{2}} \eta c_{i}^{\dagger} \exp \left(-\frac{i}{t_{0}} \boldsymbol{\lambda}^{(\alpha)} \cdot \boldsymbol{\sigma}-i a \mathcal{A}_{j i}\right) c_{j},
$$

up to $O\left(\left(\lambda_{\alpha} / t\right)^{2}\right)$ in the exponent.

In order to focus on the long-time and long-wavelength fluctuations, we take the continuum limit. In that approximation the action for the conduction electron is given by

$$
S_{c}=\int d^{3} x \bar{\psi}(x) G^{-1}\left(\left\{\hat{k}_{\mu}-\mathcal{A}_{\mu}\right\}\right) \psi(x),
$$

where $\hat{k}_{\mu}=-i \partial_{\mu}$,

$$
G^{-1}\left(\left\{k_{\mu}\right\}\right)=\left(k_{0}-t_{0} \eta \mathbf{k}^{2}\right) \sigma_{0}-\mathbf{g}(\mathbf{k}) \cdot \boldsymbol{\sigma},
$$

with

$$
\mathbf{g}(\mathbf{k})=\left(2 \eta \sum_{\alpha=x, y} k_{\alpha} \lambda_{x}^{(\alpha)}, 2 \eta \sum_{\alpha=x, y} k_{\alpha} \lambda_{y}^{(\alpha)}, \frac{J_{H}}{4}\right)
$$

and $\mathcal{A}_{\mu}=-i \bar{U} \partial_{\mu} U \equiv \Sigma_{a=x, y, z} \mathcal{A}_{\mu}^{a} \sigma_{a}$ is the $\mathrm{SU}(2)$-gauge field that describes the fluctuation of the core spins.

The derivation of the induced Chern-Simons term at the one-loop level by the derivative expansion is presented in Refs. 16, 17, and 18. The induced Chern-Simons term is given by

$$
S_{\mathrm{CS}}=-\frac{\theta}{4 \pi} \int d^{3} x \epsilon^{\mu \nu \lambda} \mathcal{A}_{\mu}^{z} \partial_{\nu} \mathcal{A}_{\lambda}^{z}
$$

where

$$
\theta=\frac{1}{4 \pi} \int d^{2} \mathbf{k} \frac{\mathbf{g}(\mathbf{k}) \times\left[\partial_{k_{x}} \mathbf{g}(\mathbf{k}) \times \partial_{k_{y}} \mathbf{g}(\mathbf{k})\right]}{|\mathbf{g}(\mathbf{k})|^{3}} .
$$

Note that only the Abelian Chern-Simons term appears in Eq. (11) because the $\mathrm{SU}(2)$-gauge field $\mathcal{A}_{\mu}$ is the pure gauge field and it satisfies the curl-free condition $\partial_{\mu} \mathcal{A}_{\nu}-\partial_{\nu} \mathcal{A}_{\mu}$ $-i\left[\mathcal{A}_{\mu}, \mathcal{A}_{\nu}\right]=0$. The non-Abelian Chern-Simons term is reduced to the Abelian Chern-Simons term upon using the curl-free condition. ${ }^{16}$ Substituting Eq. (10) into Eq. (12), we obtain

$$
\theta=\frac{1}{2} \operatorname{sgn}\left(J_{H} \Lambda\right),
$$

where $\Lambda=\lambda_{x}^{(x)} \lambda_{y}^{(y)}-\lambda_{y}^{(x)} \lambda_{x}^{(y)}$. Note that the sign of $\theta$ is determined by the sign of $\Lambda$ and $J_{H}$. The value of $\theta= \pm 1 / 2$ does not alter the statistics of particles. However, the gauge field $\mathcal{A}_{\mu}^{z}$ cannot be wiped out by an additional fermion to fermion mapping by attaching an even number of fluxes to the conduction electrons. ${ }^{19}$ Because such transformation introduces the fictitious "magnetic" field, which cannot completely cancel out $\left\langle\nabla \times \mathcal{A}_{\mu}^{z}\right\rangle$. We can extend the above calcu- 
lation to the finite temperature. ${ }^{18}$ In that case, the result is modified as $\theta= \pm \frac{1}{2} \times \tanh \left(\beta J_{H} / 8\right)$. However, if we concentrate on the region $k_{B} T \ll J_{H}$, we can neglect the finite temperature correction. Since the spin-orbit coupling term (2) involves a process of hopping between different orbitals at the same site, the external gauge field $A_{\mu}^{\mathrm{ext}}$ does not couple to it. Therefore, there is no Chern-Simons term for $A_{\mu}^{\text {ext }}$.

Now we discuss the effect of the Chern-Simons term. We take

$$
S=S_{c}+S_{\mathrm{CS}}+S_{\text {spin }}
$$

for the effective action. The last term is the action for the core-spin system and is given by

$$
\begin{aligned}
S_{\text {spin }}= & \int d t \int d^{2} \mathbf{r}\left[\bar{z}(\mathbf{r}, t)\left(i \partial_{t}-\mathcal{A}_{t}^{z}\right) z(\mathbf{r}, t)\right. \\
& \left.+J S \eta\left|\left(-i \nabla+\mathcal{A}^{z}\right) z(\mathbf{r}, t)\right|^{2}\right] .
\end{aligned}
$$

In the core-spin system, the spatial components of the gauge fields $\mathcal{A}_{\mu}^{z}$ are introduced by a Stratonovich-Hubbard transformation and the time component of that is introduced as a Lagrange multiplier field, which enforces the constraint $\bar{z}(\mathbf{r}, t) z(\mathbf{r}, t)=2 S / a^{2} .{ }^{20}$ Since we assume the ferromagnetic correlation between the core spins, we have not introduced the gauge field $A_{\mu}^{x, y}$, which describes the phase fluctuation of the staggered field. ${ }^{21}$

By integrating out the $\mathcal{A}_{t}^{z}$, we obtain the relation between the density of the conduction electron $\rho_{\sigma}(\mathbf{r}, t)$ with $\sigma=\uparrow, \downarrow$ and the "magnetic" field $\mathcal{B}(\mathbf{r}, t)=\partial_{x} \mathcal{A}_{y}^{z}(\mathbf{r}, t)-\partial_{y} \mathcal{A}_{x}^{z}(\mathbf{r}, t)$ :

$$
\sum_{\sigma} s_{\sigma} \rho_{\sigma}(\mathbf{r}, t)=\frac{\theta}{2 \pi} \mathcal{B}(\mathbf{r}, t),
$$

where $s_{\sigma}=1$ for $\sigma=\uparrow$ and $s_{\sigma}=-1$ for $\sigma=\downarrow$. Note that the Schwinger bosons do not appear in Eq. (16) because of the constraint. From Eq. (16), one can see that a conduction electron with $\uparrow(\downarrow)$ spin induces a (anti)skyrmion excitation in the core spin system. The skyrmion and antiskyrmion excitations introduce disorder in the core-spin system. In the small carrier concentration, we expect the Bose-Einstein condensation for the Schwinger bosons, which results in ferromagnetic long-range order in the core-spin system ${ }^{20}$ when we assume weak coupling between the layers. In the presence of ferromagnetic long-range order, the Meissner effect for the gauge field $\mathcal{A}_{\mu}^{z}$ occurs. The low-energy physics is governed by the Meissner phase and the Chern-Simons term has rather unimportant effect.

Since there is the same number of skyrmions and antiskyrmions as the number of conduction electrons, upon increasing the carrier number, ferromagnetic long-range order of core spins is destroyed. In this region, the Chern-Simons term Eq. (11) has dominant role for the low-energy behavior and it leads to a pairing state between conduction electrons. Coupling between the conduction-electron current and the gauge field $\mathcal{A}_{\mu}^{z}$ is given by

$$
S_{j-\mathcal{A}}=\sum_{\sigma=\uparrow, \downarrow} \int d t \int d^{2} \mathbf{r} s_{\sigma} \mathbf{j}_{\sigma}(\mathbf{r}, t) \cdot \mathcal{A}^{z}(\mathbf{r}, t),
$$

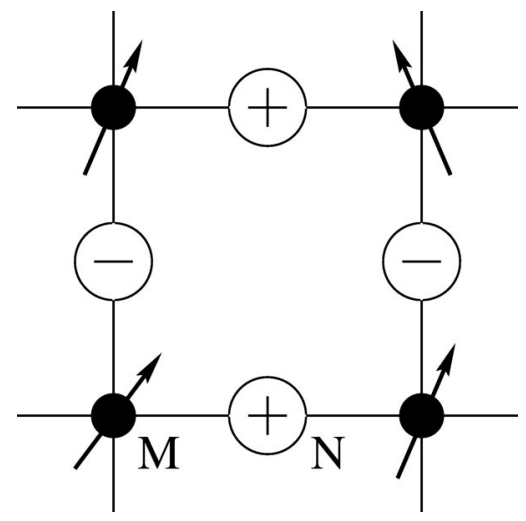

FIG. 1. Example of the system with $\Lambda \neq 0$. The filled circles represent $M$ atoms and the open circles represent $N$ atoms. Arrows denote core spins. The symbol + and - represent that $N$ atoms are displaced above and below the plane, respectively.

where $j_{\sigma}(\mathbf{r}, t)$ is the conduction electron current for $\sigma$ spin. Since Eq. (17) describes minimal coupling between the conduction current and the gauge field $\mathcal{A}^{z}$, it gives rise to a Lorentz force. Such Lorentz force is induced between each conduction electron and other conduction electrons passing by the former. Depending on the sign of the relative angular momentum, the Lorentz force becomes attractive or repulsive and leads to the chiral pairing state. From Eqs. (16) and (17), we obtain the interaction term. ${ }^{9,22}$. From that we obtain the gap equation by taking the standard procedure: ${ }^{9,22}$

$$
\Delta_{\sigma_{1} \sigma_{2}}^{\mathbf{k}}=-\frac{1}{\Omega} \sum_{\mathbf{k}^{\prime}(\neq \mathbf{k})} \frac{2 \pi i}{m_{b}} \frac{s_{\sigma_{1}} s_{\sigma_{2}}}{\theta} \frac{\mathbf{k} \times \mathbf{k}^{\prime}}{\left|\mathbf{k}-\mathbf{k}^{\prime}\right|^{2}} \frac{\Delta_{\sigma_{1} \sigma_{2}}^{\mathbf{k}^{\prime}}}{E_{\mathbf{k}^{\prime}}},
$$

where $\Delta^{\mathbf{k}}$ is the pairing matrix, $E_{\mathbf{k}}$ is the quasiparticle energy, and $m_{b} \sim 1 /\left(2 t_{0}\right)$ is the band mass of conduction electron. The gap equation (18) has a similar form as that discussed in the $\nu=1 / 2$ quantum-Hall context. ${ }^{9,22}$ The only difference is that the pairing interaction depends on the spins of conduction electrons. Following the analysis of Refs. 9 and 22 , we can show that the ground state is the $p$-wave pairing state. The pairing matrix is given by

$$
\Delta^{\mathbf{k}}=\Delta_{k}\left(\begin{array}{ll}
p e^{ \pm i \theta_{\mathbf{k}}} & q e^{\mp i \theta_{\mathbf{k}}} \\
q e^{\mp i \theta_{\mathbf{k}}} & p e^{ \pm i \theta_{\mathbf{k}}}
\end{array}\right),
$$

where $\Delta_{k}$ only depends on $k=|\mathbf{k}|, \theta_{\mathbf{k}}=\tan ^{-1}\left(k_{y} / k_{x}\right)$, and $p$ and $q$ are constants. The chirality of the pairing state (the sign in the exponent) is determined by the sign of $\theta$. The pairing state stabilized in the bulk is that with $p=0$ and $q$ $=1$, because in that case the spin of the Cooper pair lies in the plane. In the $d$-vector description, this pairing state is described by $\mathbf{d}_{\mathbf{k}}=\left(k_{x} \pm i k_{y}\right) \hat{z}$. Incidentally, this is the same as that proposed in the pairing state for the $\mathrm{Sr}_{2} \mathrm{RuO}_{4}{ }^{6,7}$

Contrary to the usual spin-fluctuation, ${ }^{23}$ which may be characterized by the Maxwell term: $\sim-\frac{1}{4}\left(\partial_{\mu} \mathcal{A}_{\nu}^{z}-\partial_{\nu} \mathcal{A}_{\mu}^{z}\right)^{2}$, in the gauge-field description, our spin fluctuation is characterized by the Chern-Simons term. Since there is an extra derivative for the former comparing with the latter, our mechanism is more dominant in the long wavelength and the 
low-energy limit than the usual spin-fluctuation mechanism. Moreover, the Chern-Simons term only exists at $2+1$ dimension. Therefore, our spin fluctuation is unique to the 2 +1 dimension. Meanwhile, the Maxwell term exists in any dimension.

For our pairing mechanism the nonvanishing value of $\Lambda$ is essential. There are several ways to realize it. An example is shown in Fig. 1, where $M$ atoms and $N$ atoms constitute the layer. The $M$ atoms consist of a core spin and a conduction orbital. Orbitals of the $N$ atoms constitute multibands and the $N$ atoms are displaced from the layer as shown in Fig. 1. The calculation for $s$ orbital for the conduction orbital of $M$ atoms and $p$ orbital for $N$ atoms is given in Ref. 11. For other possibilities of $\Lambda \neq 0$, multiband conduction electrons, the next-nearest neighbor hopping term, optical phonon modes, etc. might have some role.

To summarize, we have proposed a mechanism of uncon- ventional superconductivity in a two-dimensional doubleexchange system with spin-orbit coupling. We have shown in the presence of spin-orbit coupling the Chern-Simons term for the gauge field, which describes the phase fluctuation of the core spins, is induced. This Chern-Simons term leads to the skyrmion and antiskyrmion excitations. After magnetic long-range order is destroyed, it leads to the $p$-wave pairing between the conduction electrons.

One of the most interesting extensions of our model is the application to the antiferromagnetic core spins. In that case we need to investigate the effect of the $A, B$-sublattice, or isospin degrees of freedom. It will be discussed in future publication.

The author would like to thank M. Sigrist, K. Ohgushi, J. Goryo, A. Furusaki, and K. K. Ng for helpful discussions. This work was supported by a Grant-in-Aid from the Japanese Ministry of Education, Science, Sports, and Culture.
${ }^{1}$ P.W. Anderson, Science 235, 1196 (1987).

${ }^{2}$ Y. Maeno et al., Nature (London) 372, 532 (1994).

${ }^{3}$ T.C. Gibb et al., J. Solid State Chem. 11, 17 (1974).

${ }^{4}$ T. Oguchi, Phys. Rev. B 51, 1385 (1995).

${ }^{5}$ I.I. Mazin and D.J. Singh, Phys. Rev. B 56, 2556 (1997).

${ }^{6}$ T.M. Rice and M. Sigrist, J. Phys.: Condens. Matter 7, L643 (1995)

${ }^{7}$ G. Baskaran, Physica B 223\&224, 490 (1996).

${ }^{8}$ M. Sigrist et al., Physica C 317-318, 134 (1999).

${ }^{9}$ M. Greiter, X.G. Wen, and F. Wilczek, Nucl. Phys. B 374, 567 (1992).

${ }^{10}$ R.B. Laughlin, Science 242, 525 (1988).

${ }^{11}$ T. Ando, Phys. Rev. B 40, 5325 (1989).

${ }^{12}$ N.E. Bonesteel, T.M. Rice, and F.C. Zhang, Phys. Rev. Lett. 68, 2684 (1992); L. Shekhtman, O. Entin-Wohlman, and A. Aharony, ibid. 69, 836 (1992); W. Koshibae, Y. Ohta, and S. Maekawa, Phys. Rev. B 47, 3391 (1993).
${ }^{13}$ I. Dzyaloshinskii, J. Phys. Chem. Solids 4, 241 (1958); T. Moriya, Phys. Rev. 120, 91 (1960).

${ }^{14}$ D.P. Arovas and A. Auerbach, Phys. Rev. B 38, 316 (1988).

${ }^{15}$ E. Dagotto, E. Fradkin, and A. Moreo, Phys. Rev. B 38, 2926 (1988).

${ }^{16}$ Z. Hlousek, D. Sénéchal, and S.-H.H. Tye, Phys. Rev. D 41, 3773 (1990).

${ }^{17}$ K. Ishikawa and T. Matsuyama, Nucl. Phys. B 280, 523 (1987); Z. Phys. C 33, 41 (1986).

${ }^{18}$ K.S. Babu, A. Das, and P. Panigrahi, Phys. Rev. D 36, 3725 (1987).

${ }^{19}$ Y. Ren and F.C. Zhang, Phys. Rev. B 49, 1532 (1994).

${ }^{20} \mathrm{~A}$. Auerbach, Interacting Electrons and Quantum Magnetism (Springer-Verlag, New York, 1994).

${ }^{21}$ C. Mudry and E. Fradkin, Phys. Rev. B 49, 5200 (1994).

${ }^{22}$ T. Morinari, Phys. Rev. B 62, 15903 (2000).

${ }^{23}$ P.W. Anderson and W.F. Brinkman, Phys. Rev. Lett. 30, 1108 (1973). 